\title{
Escola de postura apoiada na tecnologia assistiva e na terapia cognitiva-comportamental em dor lombar crônica
}

Reconhecidamente de alta incidência, importante morbidade e com recidiva frequente, a dor lombar se faz presente no cotidiano de uma grande parcela da população mundial. De etiologia variada e com consequências físicas, psicoemocionais e sociais, a lombalgia crônica requer atenção ampliada, diante do seu tratamento. Para este estudo, a metodologia utilizada é de um ensaio clínico aleatório, com objetivo geral de implementar o conceito de Escola de Postura, utilizando Tecnologia Assistiva com ênfase em modelo cognitivo para o tratamento de quadros crônicos de lombalgias. A amostra foi de 20 participantes com dor lombar crônica, divididos em Grupo Experimental (GE) e Grupo Controle (GC), com alocação aleatória. Utilizaram-se instrumentos de avaliação nos períodos anterior e posterior a aplicação da abordagem experimental. A abordagem cognitiva foi aplicada no $G E$ através de aulas virtuais relacionadas a aspectos cognitivos da lombalgia crônica. A análise dos dados apoiou-se nos escores das médias das avaliações, e foram discutidos relacionando seus resultados, que se apresentaram relevantes, porém não significativos, com redução das médias das variáveis no GE. Conclui-se, então, neste trabalho, que a implementação da Escola de Postura, com enfoque cognitivo através da Tecnologia Assistiva obteve resultados positivos para o GE nos indicadores dos aspectos psicossociais e físicos.

Palavras-chave: Escola de Postura; Tecnologia Assistiva; Terapia Cognitiva-Comportamental.

\section{Posture school based on assistive technology and cognitive behavioral therapy in chronic low back pain}

\begin{abstract}
Admittedly of high incidence, important morbidity and frequent recurrence, low back pain is present in the daily lives of a large portion of the world population. Of varied etiology and with physical, psycho-emotional and social consequences, chronic low back pain requires broad attention, given its treatment. For this study, the methodology used is a randomized clinical trial, with the general objective of implementing the concept of School of Posture, using Assistive Technology with emphasis on cognitive model for the treatment of chronic low back pain. The sample consisted of 20 participants with chronic low back pain, divided into Experimental Group (EG) and Control Group (CG), with random allocation. Evaluation instruments were used before and after the application of the experimental approach. The cognitive approach was applied in EG through virtual classes related to cognitive aspects of chronic low back pain. Data analysis was based on the average scores of the evaluations, and were discussed relating their results, which were relevant, but not significant, with a reduction in the means of the variables in the EG. It is concluded, then, that the implementation of the Posture School, with a cognitive approach through Assistive Technology obtained positive results for the $E G$ in the indicators of psychosocial and physical aspects.
\end{abstract}

Keywords: Posture School; Assistive technology; Cognitive Behavior Therapy.

Topic: Fisioterapia

Reviewed anonymously in the process of blind peer.
Received: 05/03/2019

Approved: 07/05/2019
Sandro Emílio Vieira

Universidade Católica de Petrópolis, Brasil http://lattes.cnpq.br/9794817143053825 sandro.vieira@ucp.br

José Carlos Tavares da Silva (iD

Universidade Católica de Petrópolis, Brasil http://lattes.cnpq.br/3751009080198766 http://orcid.org/0000-0001-8880-2988 carlos.tavares@ucp.br
Referencing this:

VIEIRA, S. E.; SILVA, J. C. T.. Escola de postura apoiada na tecnologia assistiva e na terapia cognitiva-comportamental em dor lombar crônica. Scire Salutis, v.9, n.2, p.9-18, 2019. DOI: http://doi.org/10.6008/CBPC2236-9600.2019.002.0002 


\section{INTRODUÇÃO}

Decorrentes de várias causas nas mais diferentes idades, as lombalgias estão presentes no cotidiano de todo profissional da área da saúde. Condições que vão desde lesões ou patologias próprias da coluna vertebral até aspectos comportamentais, passando ainda por condições sociodemográficas e/ou laborais, frequentemente estão presentes de forma conjunta nos quadros clínicos dos portadores de dor lombar. $\mathrm{Na}$ sua fase aguda, a dor é consequência de uma lesão tecidual, com tempo de duração e localização desta bem delineada pela própria lesão. Contudo, o que norteia este trabalho, são as intercorrências que a dor crônica proporciona na vida de quem a sofre, mais especificamente nos indivíduos portadores de lombalgias recorrentes.

De difícil definição, a dor crônica, segundo Bastos et al. (2007), é resultante da falha nos sistemas orgânicos de garantir a homeostase do mesmo após uma lesão. Afirmam também, que a dor crônica é uma doença debilitante com consequências importantes para os estados físico, emocional e comportamental do indivíduo que a apresente. Bertor et al. (2013) enfatiza que prejuízos funcionais e psicossociais são frequentemente associados a lombalgia crônica.

Ao buscar a admissão da dor crônica como uma entidade subjetiva, entende-se que na dor crônica a relação corpo-mente está fortemente presente, pois pulsa entre estas duas vertentes, sem preferência. Gosling et al. (2011) consideram 03 dimensões para a percepção da dor: sensitivo-discriminativa (percepção e sensibilidade do estímulo doloroso quanto sua localização, intensidade, duração e qualidade), afetivoemocional (relação da dor com a emoção) e cognitivo-avaliativa (onde representa-se o comportamento, o entendimento, a memória diante da dor e a capacidade de se adaptar, com tolerância ou não, diante este estimulo doloroso).

Neste ponto, trazendo luz ao nosso trabalho, Waddel (1987) e Hoffman et al. (2007) nos dizem que a incapacidade natural apresentada pelos indivíduos com lombalgias crônicas decorre de fatores somáticos, psicológicos e sociais. E para resultados diferentes, são necessários abordagens diferentes. Como demonstrado até aqui, é imperativa a atenção ampliada ao portador de lombalgia crônica, fato justificado por este individuo apresentar quadro que vai além das implicações físicas.

Para isto, apresentaremos a Terapia Cognitiva-Comportamental (TCC) como instrumento a ser utilizado na atenção ao indivíduo com lombalgia crônica, juntamente com seu tratamento convencional. Apoiada nos pensamentos do indivíduo e a forma como este interpreta o mundo (BAHLS et al., 2004), a TCC pode se fazer importante para o indivíduo com dor lombar. Para Wright et al. (2008), a TCC fundamenta-se em duas áreas da psicologia: a Teoria Cognitiva e a Terapia Comportamental. Cada uma contribuindo com seus conceitos e experimentos, faz a sustentação teórica da TCC e a abordagem prática do tratamento.

Reconhecida por Oliveira et al. (2013) como "uma abordagem de intervenção semiestruturada, objetiva, que busca identificar e corrigir as condições que favorecem o desenvolvimento e manutenção das alterações cognitivas e comportamentais", a TCC ainda está fortemente relacionada com o sistema de crenças do indivíduo, visto que este sistema age nos sentimentos e comportamentos do mesmo. "Processos 
conscientes adaptativos de pensamento". O desenvolvimento e a utilização deste conceito é o que Wright et al. (2008) reconhecem e incentivam na TCC, focando para a obtenção da solução de problemas e de uma racionalidade do pensamento.

Oliveira et al. (2013) diz, também, que nas abordagens da TCC, busca-se auxiliar o indivíduo a identificar e alterar seus pensamentos mais elaborados, estando em destaque os pensamentos associados com aspectos psicossociais como ansiedade e depressão. Buscar unidade de abordagem na TCC é algo não conseguido, pois a diversidade é uma característica desta terapia. Porém, todas as diferentes abordagens da TCC compartilham a ideia central de que, para se avançar na terapêutica, são necessárias mudanças nos modos disfuncionais do pensamento (BAHLS et al., 2014).

Bahls et al. (2014) explica que, nos conceitos da TCC, todos os ambientes em que se vive, ofertam eventos constantemente, sofrendo estas classificações de acordo com a avaliação cognitiva, apresentandose então como positivos, negativos ou neutros. A partir desta avaliação é que se classifica então os eventos e a resposta é elaborada na forma de sentimentos ou de comportamentos. Sendo assim, os pensamentos dos indivíduos e a maneira como o mundo em que se vive é enxergado, recebe valorização e é enfatizado na TCC. E mais, o sofrimento do indivíduo devido suas distorções na autoavaliação e na avaliação do mundo é de onde se parte a abordagem da TCC.

Kamper et al. (2015) associa frequentemente a dor lombar crônica com a perda da funcionalidade e com experiências de ansiedade e depressão, além de efeitos sobre a vida laboral, social ou recreacional. E aqui percebemos as disfunções de pensamentos, facilitadas pela cronicidade do quadro, interferindo e facilitando o surgimento dos aspectos psicossociais. Como consequência deste raciocínio, Henschke et al. (2010) indica que a abordagem comportamental é comumente utilizada no tratamento da dor lombar crônica, para reduzir os efeitos desta através de modificação de comportamentos de dor mal adaptativas e processos cognitivos.

Sendo assim, esquemas, crenças e pensamentos automáticos alicerçam as emoções e comportamentos do indivíduo. E quando estes se mostram disfuncionais, emoções e comportamentos negativos se fazem presente. E neste momento a TCC, com suas mais diversificadas formas de abordagens, é uma ferramenta eficaz e objetiva na ajuda dispensada ao indivíduo.

Dentre inúmeras abordagens da TCC, optou-se aqui por utilizar estratégias de educação em saúde a atenção ao portador de lombalgia crônica. No que se refere à Educação em Saúde, esta ultrapassa os limites de um mero instrumento de promoção, indo além desta perspectiva, pois estimula o autoconhecimento e a responsabilidade que cabe ao indivíduo diante seu quadro (JANINE et al., 2015). Na prática, a Educação em Saúde para aplicação dos conceitos da TCC neste trabalho foi realizada através de duas estratégias que se complementaram: Escola de Postura e Tecnologia Assistiva. E nos parágrafos seguintes conceituaremos ambas estratégias.

Originalmente denominada 'Back School', a abordagem do tipo Escola de Postura teve sua criação em 1969, pela fisioterapeuta Mariane Zachrisson-Forssel na Suécia. Para Andrade et al. (2005), a 'Back School' consiste num 'método de treinamento postural utilizado na prevenção e tratamento de pacientes 
com dor lombar, composto de informações teórico-educativas, contendo prática de exercícios terapêuticos para a coluna'.

O objetivo principal da Escola Postural Sueca foi educar e aconselhar ergonomicamente a postura de indivíduos com dores nas costas, capacitando-os a se protegerem ativamente de suas afecções da coluna vertebral (NOLL et al., 2013). No Brasil, a primeira 'Back School' foi introduzida no Hospital do Servidor Público Estadual de São Paulo, em 1972 por Knoplich, onde recebeu o nome de 'Escola de Postura'. Na década de 1990, a Universidade Federal da Paraíba, a Escola Paulista de Medicina (Universidade Federal de São Paulo) e a Faculdade de Medicina da Universidade de São Paulo também criaram seus modelos de "Escola de Postura" (ANDRADE et al., 2005).

Abordando a efetividade das Escolas de Posturas, numa revisão sistemática de 2017, Parreira et al. concluem que há baixa evidência na qualidade das Escola de Posturas no que concerne a dor crônica devido a não padronização das abordagens aplicadas em cada projeto. Contudo, indicam que estudos futuros por apresentarem variantes diferentes podem concluírem por resultados também diferentes. Noll et al. (2013) indica que os resultados encontrados em pesquisas no Brasil referentes a eficácia de programas de 'Escola de Postura' são questionáveis, fato este identificado pela baixa qualidade metodológica dos estudos e à falta de um número maior de publicações que avaliem os resultados. Porém, reconhecem que num período imediato a abordagem há melhora dos dados pesquisados.

Nogueira nos relata que a Escola de Postura traz para sua prática aspectos sociopsicossomáticos para o portador de lombalgia crônica, além dos aspectos físicos conhecidos. O Hospital das Clínicas da Faculdade de Medicina da Universidade de São Paulo, através da Escola de Postura da Divisão de Medicina de Reabilitação, tem como objeto norteador o entendimento da utilização da educação para prevenção e tratamento de quadros clínicos de lombalgia.

Incentiva-se o indivíduo quanto a sua responsabilidade diante do seu quadro, fazendo isto a partir do momento em que direciona sua atenção a aspectos de toda natureza do cotidiano que influencia a dor na região lombar e ao proporcionar, através do conteúdo cognitivo-comportamental passado nas estratégias da Escola de Postura, formas de contornar os fatores do quadro álgico e facilitar o controle deste sintoma (TSUKIMOTO et al., 2006).

No que concerne a Tecnologia Assistiva (TA), Rocha et al. (2012) destaca que na literatura internacional as definições à cerca de Tecnologia Assistiva estão voltadas para a utilização de recursos, estratégias e serviços que são aplicados para minimizar as alterações funcionais de um indivíduo. E assim, permitem ou ampliam as habilidades deste indivíduo. Como consequência, a TA visa proporcionar a esta independência, qualidade de vida e inclusão. Corroborando com o aqui exposto, a TA apresenta-se como um fenômeno multidimensional, onde abrange diversos aspectos como biomecânica, mecânica, tecnologia, ética, afeto, cognição, ergonomia, estética e outros (ROCHA et al., 2005). A associação entre um modelo de Escola de Postura com as ferramentas da TA poderá proporcionar avanço cognitivo ao indivíduo acometido por lombalgia crônica. 


\section{METODOLOGIA}

A proposta metodológica deste trabalho consiste em ensaio clínico aleatório e como procedimento para a garantia do aspecto ético, o projeto acerca desta pesquisa foi devidamente registrado na Plataforma Brasil, sendo submetido ao Comitê de Ética na Pesquisa da Universidade Católica de Petrópolis (CEP/UCP) e aprovado pelo mesmo no dia 15 de junho do ano de 2016. A assinatura do Termo de Consentimento Livre e Esclarecido, modelo CEP/UCP foi obrigatória para todos os participantes da pesquisa.

A amostra foi composta por indivíduos que se enquadraram nos critérios definidos para a pesquisa. Estes critérios versavam sobre indivíduos que apresentavam quadro álgico, do tipo inespecífica, da região lombar da coluna vertebral por mais de 03 meses consecutivos, indicação médica para tratamento fisioterapêutico e ainda, no mínimo, ter histórico de mais um episódio de natureza igual ou semelhante ao apresentado no momento. Idade, escolaridade e intensidade de dor no momento da anamnese não excluíam o participante. Contudo, um quadro de dor lombar classificado como agudo no processo de avaliação seria o critério de exclusão da pesquisa.

No que se refere a abordagem fisioterapêutica ofertada para a amostra, elaborou-se o Plano Cinesioterapêutico para Lombalgia Crônica que consistia em exercícios de alongamento e fortalecimento muscular e exercícios respiratórios para relaxamento. O Grupo Controle (GC) foi submetido aos instrumentos de avaliação selecionados para este trabalho, e que serão apresentados na sequência deste, no início do experimento. $O$ tratamento fisioterapêutico realizado pelo participante deste $G C$ foi mantido até a reavaliação do mesmo, sendo aplicadas 10 sessões do Plano Cinesioterapêutico citado no parágrafo acima.

No que se refere ao Grupo Experimental (GE), os participantes realizaram o protocolo do Plano Fisioterapêutico proposto, contudo acrescentou-se um programa teórico-cognitivo da 'Escola de Postura', com uso da Tecnologia Assistiva. Este programa consistiu de 05 apresentações, em grupo ou individualmente, de aulas virtuais que abordavam os seguinte temas: Ansiedade e dor nas costas; Orientações para atividades domésticas sem comprometer a coluna vertebral; A importância dos cuidados com a coluna vertebral ; Doenças Psicossomáticas (para este assunto foram utilizados 02 vídeos).

Após o contato inicial com cada participante, procedeu-se, então, de forma aleatória a escolha do grupo ao qual o indivíduo seria alocado. A pesquisa iniciou-se com 30 indivíduos, porém ao completar-se o período da coleta de dados 10 participantes abandonaram a mesma, por motivos pessoais ou sem justificativa apresentada. Sendo então coletados os dados completos de 20 participantes. Para a amostra final, dos 20 indivíduos participantes 11 se declararam do sexo feminino e 09 do sexo masculino. 0 integrante da pesquisa mais jovem indicava 21 anos e 79 anos foi a participante de maior idade. E ainda, a média de idade apresentada foi de aproximadamente 56 anos.

Para as medidas pré e pós-experimento, utilizou-se primeiramente o modelo atual de anamnese instituído pelo curso de Fisioterapia da Universidade Católica de Petrópolis, a Escala Visual Analógica de dor (MAGGE, 2002), o Questionário de Incapacidade Funcional de Roland-Morris, o Inventário de Depressão de Beck, o Inventário de Ansiedade de Beck e a Escala de Desesperança de Beck. 


\section{RESULTADOS E DISCUSSÃO}

Como alternativa de quantificação dos dados, entendemos, neste trabalho, que as variáveis se apresentam nas seguintes formas: percepção subjetiva da dor, incapacidade física para atividades diárias por cauda do quadro álgico lombar, depressão, ansiedade e desesperança. E para facilitar a observação dos resultados, apresentamos, aqui, quadros referentes a estes tanto para o GC quanto para o GE.

Quadro 1: Percentis das médias das avaliações do GC.

\begin{tabular}{|l|c|c|c|}
\hline Variável & Pré-experimento & Pós-experimento & Variação do Percentil \\
\hline Dor & 5,8889 & 5,8889 & $0 \%$ \\
\hline Incapacidade & 112,333 & 112,556 & $1,80 \%$ \\
\hline Depressão & 9,5556 & 11,444 & $19,76 \%$ \\
\hline Ansiedade & 13,667 & 14,5556 & $6,50 \%$ \\
\hline Desesperança & 3,7778 & 3,7778 & $0 \%$ \\
\hline
\end{tabular}

Quadro 2: Percentis das médias das avaliações do GE.

\begin{tabular}{|l|c|c|c|}
\hline Variável & Pré-experimento & Pós-experimento & Variação do Percentil \\
\hline Dor & 5,3636 & 4,2727 & $18,019 \%$ \\
\hline Incapacidade & 12,455 & 9,1818 & $18,557 \%$ \\
\hline Depressão & 10,364 & 7,2727 & $33,097 \%$ \\
\hline Ansiedade & 15,909 & 11,364 & $27,26 \%$ \\
\hline Desesperança & 3,5455 & 3 & $15,38 \%$ \\
\hline
\end{tabular}

E assim, apresentamos os dados obtidos sobre nossas variáveis, o que nos subsidiam agora para avançarmos na discussão do nosso trabalho. Para Rocha et al. (2005), o eixo responsável pela centralização da TA é a relação entre indivíduo e tecnologia. Nessa relação, é de responsabilidade da tecnologia empregada melhorar ou manter as habilidades e funções do indivíduo assistido.

E aqui iniciamos nossa discussão do trabalho, pois concordando com a afirmação acima, buscamos a ferramenta da TA para intervir no grupo experimental da nossa amostra, ansiando para que através desta dualidade, indivíduo/tecnologia, a oferta cognitiva do nosso tema fosse absorvida pelos participantes e nos trouxesse respostas comportamentais positivas diante da lombalgia.

Definidas nossas variáveis, percorremos, então, o caminho para as suas avaliações. Para isso, assim como Cruz et al. (2003), focamos na multidimensionalidade do fenômeno da dor lombar, de classificação crônica. E nesse contexto, é necessário identificar a presença de aspectos emocionais e/ou comportamentais interferindo de forma a maximizar as manifestações dolorosas e incapacitantes da lombalgia.

Para cada variável apresentada, uma avaliação referendada foi aplicada. Pois além da quantificação e da interpretação da dor, o indivíduo revelava aspectos subjetivos desta, a interferência desta no seu cotidiano e ainda, as suas sensações diante deste sintoma associado as suas autopercepções de desesperança, ansiedade e depressão.

Neste momento do trabalho, ao desviarmos nosso olhar para o item da dor, começamos nosso diálogo com as médias desta variável. No período pré-experimento, para nosso GC encontramos escore médio de 5,8889, representatividade esta da subjetividade da dor. E percebemos, ainda neste mesmo grupo, porém agora no período pós-experimento, que a média não se alterou, permanecendo o mesmo valor de 5,8889 . No que concerne ao GE, a média inicial da EVA (pré-experimento) apresentada foi de 5,3636, ficando 
muito próxima da média inicial do GC. E ao avançarmos para a avaliação desta variável no período após a intervenção experimental da abordagem cognitiva, o valor da média da EVA foi de 4,2727.

Apoiando-nos na demonstração percentual desta variável, observamos nenhuma variação para o grupo controle, com ele se mostrando rigorosamente igual nas suas médias pré e pós-experimento. Mas aqui trazemos o dado, ligado GE, que para este grupo houve variação das médias nos períodos anterior e posterior ao experimento representou uma redução em seus valores na ordem de $18,019 \%$. Ou seja, para o GE a percepção subjetiva da dor, medida através da EVA de Magee (2002), após a abordagem cognitiva auxiliada pela estratégia da TA, apresentou-se menor.

Assim como Tsukimoto et al. (2006) concluiu em trabalho relacionado a Escola de Postura e dor lombar que a percepção da dor é reduzida quando se aborda a mesma, conseguimos, aqui neste ensaio, a redução da dor para o nosso GE. Segundo Ostelo et al. (2005), é necessário se apoiar em um critério de mudança minimamente clinicamente importante (minimally clinically important change - MCIC), para afirmativa de uma melhora clínica dos indivíduos com lombalgia. Neste trabalho, a redução da média da sensação subjetiva da dor do GE foi de 1,0909. O que para os autores acima, encontra-se abaixo dos 2,5 pontos estipulados por estes para classificar-se como uma diminuição clinicamente satisfatória.

A variável da incapacidade funcional, analisada através do Questionário de Incapacidade de RolandMorris, também nos trouxe importante alteração nas médias do GE durante nossa pesquisa. Antes de descrevermos este grupo voltemos nossas atenções para o GC, onde foi observado uma ligeira mudança nas médias desta variável nas fases anterior e posterior ao experimento, sem consideração estatística relevante, de 1,8081\%. Mas no que se refere ao GE, a redução de 32,557\% entre as médias (pré e pós-experimento) de incapacidade funcional se mostrou relevante.

Nossa amostra, para a variável da incapacidade funcional, apresentou escore médio de 12,4, o que se aproxima muito de um estudo realizado no Reino Unido e apresentado por Santos (2015), que analisou a lombalgia crônica e aspectos psicológicos em 1591 indivíduos. Nesse estudo, a média do questionário de Roland-Morris foi 12,6 pontos, portanto similar aos nossos números. 78 participantes compuseram a amostra do estudo de Meyer e colaboradores Santos (2015) para lombalgia crônica. E para esta amostra, a média do questionário de incapacidade de Roland-Morris também se apresenta em consonância com a nossa média, nos trazendo 11,4 pontos como valor médio.

E ao vermos todas as médias aqui apresentadas para esta variável, sempre girando em torno de 12 pontos, temos a convicção de que a média do GE deste trabalho, que na avaliação pré-experimento se mostrava com 12,455 pontos, após a aplicação da estratégia cognitiva aponta para uma importante modificação, nos conduzindo para um resultado positivo, pois 9,1818 pontos representa uma vitoriosa redução nos escores de incapacidade funcional para os portadores de lombalgia crônica.

Ainda invocando o MCIC apresentado por Ostelo et al. (2005), para a avaliação de Roland-Morris sobre incapacidade, nossos dados ficaram ligeiramente abaixo do escore indicado pelos autores de 3,5 pontos para considerarmos uma alteração clinicamente satisfatória, apresentando uma redução na ordem de 3,28 pontos. Para as nossas variáveis diretamente relacionadas com o comportamento dos participantes 
da pesquisa, foi importante a constatação de que aquela relacionada a ansiedade se mostrou fortemente presente na amostra.

Considerando a classificação recomendada pelo próprio Inventário de Ansiedade de Beck, encontramos indivíduos enquadrados tanto na classificação mínima da avaliação quanto na classificação grave para esta mesma avaliação. Contudo, no GE, não foram raros os momentos em que um participante descia de patamar após a avaliação pós-experimento. Para este dado relatado, 04 participantes desceram de patamar nas suas classificações após a inserção cognitiva. Enquanto 06 reduziram seus escores, porém se mantiveram na classificação e 01 participante apresentou escore mais elevado na segunda avaliação, indicando aumento da sua classificação.

Nossos resultados demostraram uma melhora na média desta avaliação no GE, representando 27,26\% menor nas avaliações aplicadas após a abordagem cognitiva, razão do experimento. O GC, por sua vez, teve sua média aumentada entre as avaliações pré e pós-experimento, sendo este aumento aproximadamente de 6,50\%. O estudo preliminar de Cruz et al. (2003), com temática relacionada à lombalgia e aspectos emocionais, mostrou que aproximadamente $50 \%$ da população estudada apresentou alto índice para a presença de ansiedade.

Santos (2015), em sua dissertação sobre lombalgia, incapacidade funcional e aspectos psicológicos, reuniu uma amostra de 84 indivíduos divididos em grupo controle (sem lombalgia) e grupo experimental (com lombalgia). Neste seu trabalho, indica-nos que a ansiedade presente em seu grupo experimental possui indícios de relação com a cronicidade da dor e suas consequências físicas e sociais, causando no estado afetivo do portador da lombalgia crônica um sentimento de sofrimento e incerteza em relação ao seu futuro no que se relaciona com sua dor e sua capacidade funcional. A depressão é um achado psicológico de elevada significância na população, com lombalgia estudada por Cruz et al. (2003), variável esta que será abordada na sequência desta discussão.

Ao iniciarmos a coleta de dados, a média apresentada por nossa amostra foi de 10 pontos no Inventário de Depressão de Beck. Em sua dissertação, Santos (2015) apresentou trabalhos com médias para de 7,3 e 16,06 pontos para esta mesma avaliação. Contudo, no processo avaliativo pós-experimento, nossa média reduziu para 9,15 pontos. Fato este ocorrido em especial no $\mathrm{GE}$, pois para o $\mathrm{GC}$ houve uma variação para cima das médias relacionada a depressão, iniciando com 9,5556 pontos e passando para 11,444 pontos.

No que concerne ao GE, com escore de 10,364 pontos de média nas avaliações iniciais, este apresentou uma redução com percentil de 33,097\% nas avaliações aplicadas após a estratégia da Escola de Postura com a abordagem cognitiva através da TA. Apresentando 7,2727 pontos como média para o Inventário de Depressão de Beck após o experimento.

A Escala de Desesperança de Beck, instrumento utilizado para esta variável, trouxe-nos como resultados os menores escores dentre todas as nossas avaliações. Com média de 3,65 pontos no momento pré-experimento e de 3,35 após a aplicação do experimento, a desesperança em relação ao futuro dos participantes do estudo não se confirmou em estado crítico. 
O GC manteve sua média em todas as fases da pesquisa (3,7778 pontos), enquanto o GE reduziu sua média de 3,5455 pontos para 3 pontos na escala criada por Beck, o que representa queda de 15,38\%. Desta forma, apresentados e discutidos os dados da pesquisa, dialogando-se com a literatura pertinente ao assunto, encerramos este capítulo avançando para a conclusão como próximo passo.

\section{CONCLUSÕES}

Estudo experimental de característica preliminar, buscamos aqui relacionar a lombalgia crônica com aspectos emocionais representados por depressão, ansiedade e desesperança e aspectos físicos ligados a incapacidade funcional do indivíduo. Observou-se, então, com base nos resultados obtidos, não estatisticamente significativos, alterações das médias das variáveis. Ressalta-se que as disfunções emocionais estão presentes na amostra, com ênfase para as condições de ansiedade e depressão e em menor escala para a variável desesperança.

No contexto da incapacidade funcional dos indivíduos da amostra, esta não se confirma presente na amostra de forma veemente, visto que suas médias ficaram abaixo da classificação de incapacidade indicada no instrumento de avaliação para esta variável. A utilização da abordagem cognitiva para o GE foi eficaz nos índices das ferramentas avaliativas empregadas no trabalho, apresentando redução nos percentis para todas as variáveis da pesquisa.

Apoiando-se nas referências da literatura e tornando nossos dados os alicerces deste trabalho, conclui-se que uma estratégia de Escola de Postura associada com o uso de Tecnologia Assistiva mostrou-se interessante ferramenta para a oferta cognitiva ao indivíduo portador de lombalgia crônica quanto ao seu quadro clínico, tanto para as limitações funcionais e o quadro álgico como para as disfunções psicoemocionais representadas por depressão, ansiedade e desesperança.

Considerando a abrangência do tema central desta pesquisa, a lombalgia, e as infinitas possibilidades de atenção a esta, aqui representados pela Tecnologia Assistiva e pela Terapia Cognitiva-Comportamental e também suas influências nos seus portadores, se faz necessário a continuidade e ampliação dos estudos dedicados ao assunto, e mesmo assim não se esgotaram todos os aspectos relacionados a esta 'entidade'.

\section{REFERÊNCIAS}

ANDRADE, S. C.; ARAÚJO A. G. R.; VILAR, M. J. P.. 'Escola de Coluna': Revisão Histórica e Sua Aplicação na Lombalgia Crônica. Revista Brasileira de Reumatologia, v.45, n.4, p.224-228, 2005.

ANDRADE, V. S.; PEREIRA, L. S. M.. Influência da tecnologia assistiva no desempenho funcional e na qualidade de vida de idosos comunitários frágeis: uma revisão bibliográfica. Revista Brasileira de Geriatria e Gerontologia, v.12, n.1, p.113-122, 2009.

BAHLS, S. C.; NAVOLAR, A. B. B.. Terapia CognitivoComportamentais: Conceitos e Pressupostos Teóricos. Revista Eletrônica de Psicologia, Curitiba, n.4, 2004.
BASTOS, D. F.; SILVA, G. C. C.; BASTOS, I. D.; TEIXEIRA, L. A.; LUSTOSA, A. L.; BORDA, M. C. S.; COUTO, S. C. R.; VICENTE, T. A.. Dor. Revista da Sociedade Brasileira de Psicologia Hospitalar, Rio de Janeiro, v.1, n.10, 2007.

BECK, A. T.; ALFORD, B. A. O Poder Integrador da Terapia Cognitiva. Porto Alegre: Artes Médicas, 2000.

BERTOR, W. R. R.; FRACARO, G. A.; SILVA, L. I.; ZILIO, M.; ARAGÃO, F. A.; CARVALHO, A. R.. Subclassificação da lombalgia crônica e nível de incapacidade: efeito no desempenho funcional e força muscular. ConScientiae Saúde, v.12, n.4, p.563-571, 2013.

BURNS, J. W.; GERHART, J. I.; BRUEHL, S.; POST, K. M.; SMITH, D. A.; PORTER, L. S.; SCHUSTER, E.; BUVANENDRAN, 
A.; FRAS, A. M.; KEEFE, F. J.. Anger Arousal and Behavioral Anger Regulation in Everyday Life Among People with Chronic Low Back Pain: Relationships with Spouse Responsesand Negative Affect. Health Psychology, v.34, n.5, p.547-555, 2015. DOI: http://doi.org/10.1037/hea0000221

CRUZ, R. M.; SARDÁ JUNIOR, J. J.. Diagnóstico de Aspectos Emocionais Associados à Lombalgia e à Lombociática. Avaliação Psicológica, v.1, p.29-33, 2003.

GOSLING, A. P.; SILVA, C. D. C.; PÉRISSÉ, F.. Neurofisiologia da Dor. In: BACHUR, J. A.; VEZZANI, S.. Programa de Atualização em Fisioterapia Esportiva e Traumatoortopédica. Porto Alegre: Artmed, 2011. p.57-112.

HENSCHKE, N.; OSTELO, R. W. J. G.; TULDER, M. W.; VLAEYEN, J. W. S.; MORLEY, S.; ASSENDELFT, W. J. J.; MAIN, C. J.. Behavioural treatment for chronic low-back pain. Cochrane Database of Systematic Reviews, n.7, 2010.

HEYMANS, M. W.; TULDER, M. W.; ESMAIL, R.; BOMBARDIER, C.; KOES, B. W.. Back schools for non-specific low-back pain. Cochrane Database of Systematic Reviews, n.4, 2004.

HOFFMAN, B. M.; PAPAS, R. K.; CHATKOFF, D. K.; KERNS, R. D.. Meta-Analysis of Psychological Interventions for Chronic Low Back Pain. Health Psychology, v.26, n.1, p.1-9, 2007. DOI: http://doi.org/10.1037/0278-6133.26.1.1

JANINE, J. P.; BESSLER, D.; VARGAS, A. B.. Educação em saúde e promoção da saúde: impacto na qualidade de vida do idoso. Saúde Debate, Rio de Janeiro, v.39, n.105, p.480490, 2015.

KAMPER, S. J.; APELDOORN, A. T.; CHIAROTTO, A.; SMEETS, R. J. E. M.; OSTELO, R. W. J. G.; GUSZMAN, J.; TULDER, M. W.. Multidisciplinary biopsychosocial rehabilitation for chronic low back pain: Cochrane systematic review and meta-analysis. BMJ, v.350, 2015. DOI:

http://doi.org/10.1136/bmj.h444

MAGGE, D. J.. Avaliação Músculo Esquelética. 4 ed. São Paulo: Manole, 2002.
NOLL, M.; VIEIRA, A.; DARSKI, C.; CANDOTTI, C. T.. Escolas posturais desenvolvidas no Brasil: revisão sobre os instrumentos de avaliação, as metodologias de intervenção e seus resultados. Revista Brasileira de Reumatologia, v.54, n.1, p.51-58, 2013.

OLIVEIRA, L. L.; DEIRO, C. P.. Terapia Cognitivo-

Comportamental para Transtornos Alimentares: A Visão de Psicoterapeutas sobre o Tratamento. Revista Brasileira de Terapia Comportamental e Cognitiva, v.14, n.1, p.36-49, 2013.

OSTELO, R. W. J. G.; VET, H. C. W.. Clinically importante outcomes in low back pain. Best Practice \& Research Clinical Rheumatology, v.4, n.4, p.593-607, 2005.

PARREIRA, P.; HEYMANS, M. W.; TULDER, M. W.; ESMAIL, R.; KOES, B. W.; POQUET, N.; LIN, C. W. C.; MAHER, C. G.. Back schools for chronic non-specific low back pain (protocol). Cochrane Database of Systematic Reviews, n.5, 2015.

ROCHA, A. N. D.; DELIBERATO, D.. Tecnologia assistiva para a criança com paralisia cerebral na escola: identificação das necessidades. Revista Brasileira de Educação Especial, Marília, v.18, n.1, p.71-92, 2012.

ROCHA, E. F.; CASTIGLIONI, M. C.. Reflexões sobre recursos tecnológicos: ajudas técnicas, tecnologia assistiva, tecnologia de assistência e tecnologia de apoio. Revista de Terapia Ocupacional da Universidade de São Paulo, v.16, n.3, p.97-104, 2005.

SANTOS, M. C. F.. Relação da ansiedade, depressão e estresse com a incapacidade funcional em indivíduos com dor lombar. Dissertação (Mestrado) - Universidade Norte do Paraná, Londrina, 2015.

TSUKIMOTO, G. R. R., M.; BRITO, C. A.; BATTISTELLA, L. R.. Avaliação longitudinal de Escola de Postura para dor lombar crônica: através da aplicação dos questionários Roland Morris e Short Form Health Survey (SF 36). Acta Fisiátrica, v.13, n.2, p.63-69, 2006.

WRIGHT, J. H.; BASCO, M. R.; THASE, M. E.. Aprendendo a Terapia Cognitiva-Comportamental: Um Guia Ilustrado. Porto Alegre: Artmed, 2008.

A CBPC - Companhia Brasileira de Produção Científica (CNPJ: 11.221.422/0001-03) detém os direitos materiais desta publicação. Os direitos referem-se à publicação do trabalho em qualquer parte do mundo, incluindo os direitos às renovações, expansões e disseminações da contribuição, bem como outros direitos subsidiários. Todos os trabalhos publicados eletronicamente poderão posteriormente ser publicados em coletâneas impressas sob coordenação da Sustenere Publishing, da Companhia Brasileira de Produção Científica e seus parceiros autorizados. Os (as) autores (as) preservam os direitos autorais, mas não têm permissão para a publicação da contribuição em outro meio, impresso ou digital, em português ou em tradução. 